\title{
Afectividad inducida e impacto en la creatividad, crecimiento personal después del cambio y ajuste percibido al narrar una experiencia emocional intensa
}

\author{
Silvia da Costa * y Darío Páez
}

Departamento de Psicología Social y Metodología de las Ciencias del Comportamiento, Universidad del País Vasco

\begin{abstract}
Resumen: Se examinan en un estudio experimental los efectos de la inducción de afectividad positiva en la creatividad y la importancia de las emociones como variables mediadoras. Se indujo alta y baja afectividad positiva pidiendo a las personas participantes que redactaran tres poemas haikus sobre dos estímulos de connotación positiva, alta $(N=28)$ y baja $(N=28)$. Las personas escribieron sobre su experiencia de enamoramiento más in tenso y contestaron una escala sobre las emociones sentidas al redactar el texto, una medida de crecimiento personal y una tercera referida a la obtención de metas adaptativas después del episodio. En la condición de alta afectividad positiva se informó de más emociones positivas y menos negativas al narrar la experiencia, un mayor crecimiento personal y un mayor ajuste. Jueces independientes evaluaron como más originales el último poema y la narración de la experiencia en la condición de alta afectividad positiva. Un análisis mediacional mostró que las emociones positivas explicaban la influencia de la manipulación sobre el crecimiento personal y el ajuste percibido.

Palabras clave: Afectividad positiva; ajuste percibido; análisis mediacional; creatividad; crecimiento post-estrés.
\end{abstract}

"It sounded an excellent plan, no doubt, and very neatly and simply arranged. The only difficulty was, she had not the smallest idea how to set about it." Lewis Carrol, of Alice in Alice in Wonderland

\section{Introducción}

La creatividad es un aspecto fundamental de la cognición y de la conducta humana. Aunque no hay un consenso absoluto sobre lo que significa este constructo, hay quienes plantean que es una manera de hacer frente a los desafíos de la vida, diferente de la inteligencia, pero relacionada con ella (Artola et al., 2012; Mirowsky y Ross, 2007).

\section{Creatividad en términos de tres p: persona, proceso y producto creativo}

Han (2003) define la creatividad en términos de persona, proceso y producto creativos. La creatividad personal sería similar a una variable independiente que influye en la creatividad y el producto creativo sería la variable dependiente como una medida de la misma. Sternberg y Lubart (1995) han propuesto que son seis los recursos que alimentan este constructo y cinco de ellos pertenecen a la categoría de persona creativa. La correlación entre variables de personalidad con la creatividad fueron investigados en diferentes meta-análisis confirmándose que la apertura a la experiencia como rasgo de personalidad se asocia claramente a la creatividad (Ma, 2009). Otro punto de vista es el de los investigadores que se han centrado en el proceso creativo definiendo la creatividad como un proceso de percepción de pro-

* Dirección para correspondencia [Correspondence address]: Silvia da Costa. Departamento de Psicología Social y Metodología de las Ciencias del Comportamiento, Avenida de Tolosa, 70, Donostia/San Sebastián (España). E-mail: silviacristina.dacosta@ehu.es
Title: Induced affect impact on creativity, post change personal growth and perceived adjustment after narrating an intense emotional experience. Abstract: An experimental study contrast the effects of inducing positive affect on creativity and the mediational role of positive emotions. High and low positive affect was induced by asking subjects to write three poems Haikus about high $(N=28)$ and low positive connotation stimuli $(N=28)$. Then people wrote about their more intense infatuation experience and answered a scale on the emotions felt when writing the text, a measure of personal growth or benefits, and a measure of achievement of adaptive goals after the episode. In high positive affect condition was reported more positive and less negative emotions in recounting the experience, more personal growth and a better adjustment. Last poem and narration of experience were evaluated as more original in high positive condition by independent judges. A mediational analysis showed that positive emotions explain partially the influence of the manipulation on personal growth and perceived adjustment.

Key words: Positive affect; perceived adjustment; meditational analysis; creativity; post stress growth.

blemas o lagunas de información, de formación de ideas o hipótesis, de evaluar y modificar estas hipótesis y comunicar los resultados (Oliveira et al., 2009). Csikszentmihalyi (2011), que desde una perspectiva sistémica la define como cualquier acto, idea o producto que cambia un campo existente, o que transforma un campo existente en uno nuevo, introduce al hablar de proceso creativo la noción de "flujo" para referirse a la experiencia de sentirse intensamente implicado en una actividad. Algunas definiciones de creatividad se han orientado al resultado o producto creativo, conceptualizando la misma como la capacidad de producir algo nuevo y útil o de valor (Henessey y Amabile, 2010).

\section{Evaluación de la creatividad}

La respuesta y el producto creativo, en particular en la tradición de estudios psicológicos de la cognición, se evalúan en función de dimensiones, p.e., la fluidez -que se define como la cantidad de ideas y soluciones producidas en relación a problemas o tareas que se presentan-, suele evaluarse como número total de ideas dadas como solución; la flexibilidad -que se refiere a la producción de una amplia variedad de ideas o soluciones que pertenecen a categorías diferentes y la capacidad para cambiar rápidamente entre varios tipos o clases de ideas y soluciones-, suele evaluarse contando el número de categorías distintas utilizadas en las respuestas a la tarea o problema dado; la originalidad -se refiere al carácter inusual, único y poco frecuente de las ideas o soluciones-, suele evaluarse ponderando las respuestas menos frecuentes y menos usuales; la elaboración -que se refiere a la cantidad de detalles de la idea o solución, al interés y organización del producto y/o de la solución-, suele evaluarse valorando la extensión o el detalle de las ideas, soluciones o productos; la 
calidad de la solución se refiere al carácter apropiado, la eficacia y aplicabilidad real de la idea o solución (Artola et al., 2012; Ma, 2009; Runco, 1999)

Las evaluaciones de la creatividad difieren en cómo se genera el indicador, de forma que podemos distinguir tres fuentes básicas: a) auto-informes sobre las conductas de generación e implementación de ideas nuevas (por ejemplo listas de conductas o actividades creativas), b) hetero-informes o evaluaciones por supervisores y pares de las mismas conductas y c) evaluación más "objetiva” del número de ideas, del número de sugestiones, de textos o productos intelectuales, patentes y mediante la evaluación de expertos de los elementos anteriores (Ma, 2009; Hunter, Bedell y Mumford, 2007; Hammond, Neff, Farr, Schwall y Zhao, 2011). La evaluación por expertos de productos creativos (desde poemas hasta a innovaciones tecnológicas) mediante acuerdo consensual es una forma ampliamente utilizada de la creatividad (Henessey y Amabile, 2010).

\section{Afectividad positiva y creatividad}

El estudio que se presenta en este artículo examina si personas en las que se induce alta afectividad positiva presentan mayor creatividad que en aquellas que se induce baja afectividad positiva. También se buscó conocer cuál es la importancia de las emociones como variables mediadoras. Aunque hay una larga tradición de estudio de la influencia de la afectividad positiva en la creatividad (Baas, De Dreuw y Nijstad, 2008) hay pocos estudios sobre la asociación entre esta última y la afectividad positiva inducida mediante escritura poética. También hay pocos estudios sobre la relación entre episodios de cambio intenso de valencia positiva, como enamoramiento intenso, y crecimiento post-estrés (Bilbao, Páez, da Costa y Martínez-Zelaya, 2013). No hemos encontrado estudios sobre la relación entre afectividad positiva inducida y crecimiento, si hay estudios sobre atribución de significado y afectividad positiva inducida pero no en relación a crecimiento post-estrés (Fredrickson, 2009).

Las emociones sirven para modelar, cambiar y mejorar el pensamiento al dirigir nuestra atención hacia la información significativa de un modo más rápido (Salovey y Mayer, 1990); también facilitan el cambio de perspectiva, la formación de juicios y la consideración de nuevos puntos de vista de los problemas, debido a las continuas fluctuaciones emocionales (Fernández-Berrocal y Extremera, 2003). Por ejemplo, algunas emociones positivas pueden ser útiles para estimular el pensamiento creativo, facilitando el pensamiento y la capacidad de funcionamiento de las personas (Isen, 2007). Se ha planteado que la afectividad positiva está asociada a un procesamiento más creativo y heurístico mientras que la afectividad negativa estaría relacionada con un procesamiento más exhaustivo (Palfai y Salovey, 1993). Hay evidencia parcial de que las emociones positivas amplían el repertorio conductual y ayudan a manejar y reducir el impacto de las emociones negativas (Isen, 1999; Fredrickson, 2009). Se ha encontrado en un estudio (Scheier, Weintraub y Carver,
1986) que el afecto positivo y el optimismo pueden ser una fuente de auto-control y un recurso que capacita para resolver situaciones difíciles al reducir la posibilidad de estar a la defensiva y conducir a las personas a ser más abiertas y tener una mayor aceptación cuando las cosas no pueden cambiarse, pero más actividad para cambiarlas cuando esto es posible. Además, el afecto positivo promueve una razonable capacidad de ayuda, generosidad y responsabilidad social (Isen, 2007). Concluyendo, se plantea que a medio plazo, la afectividad y las emociones positivas refuerzan los recursos personales y sociales (Fredrickson, 2009).

De forma más relevante para nuestra problemática, las emociones positivas amplían el repertorio mental: el afecto activa las capacidades para la resolución creativa de problemas, induce la generación de más ideas, un uso más flexible de las categorías, guía y facilita la organización en la memoria posibilitando la eficiencia cognitiva generada por dicha organización (Isen, 2007). La afectividad positiva refuerza la motivación intrínseca, facilita el pensamiento y el juicio, capacitándolos para que sean más flexibles favoreciendo la resolución de problemas más generales. Por ejemplo, las personas con afecto positivo intentan hallar más soluciones ante problemas difíciles (Fredrickson, 2009; Isen, 2007).

La relación entre la afectividad y emociones positivas en relación a la afectividad y emociones negativas es un aspecto central del bienestar hedónico -compuesto por la afectividad positiva, negativa y la satisfacción vital- (Diener, 2009). A la relación entre ambas dimensiones se le denomina balanza de afectos. Una buena balanza de afectos implica más afectividad positiva que negativa y Fredrickson (2009) plantea que una ratio de 3 a 1 o más de emociones positivas sobre negativas permite un mejor funcionamiento psicológico, por lo que se puede suponer que a más afectividad positiva, mayor creatividad. Congruentemente con la influencia de la afectividad positiva en el pensamiento y la creatividad, el metaanálisis de Baas, De Dreu y Nijstad (2008) encontró que la afectividad positiva se asociaba a mayor creatividad que un grupo neutro $(r=.15)$. Con respecto al tipo de emociones, el incremento de la creatividad se producía en el caso de afectos positivos de alta activación (como alegría), pero no cuando la afectividad positiva era de baja activación (como calma). Comparando grupos de afecto positivo con negativo el meta-análisis de Baas et al. (2008) encontró que había diferencia en dos de cuatro aspectos de la creatividad: la afectividad positiva refuerza la originalidad y la fluidez de la respuesta creativa $(r=.11$ y $r=.09)$, aunque no tenía relación con el insight y la flexibilidad. También el tiempo de inducción de afecto influía. El efecto disminuía a medida que el tiempo de tarea era mayor. Finalmente, había un efecto de congruencia entre el tipo de tarea y la influencia de la afectividad positiva. El efecto de aumento de la creatividad se daba cuando la afectividad era positiva y la tarea se enmarcaba de esta forma, era de ganancia e intrínsecamente interesante. Por el contrario, la afectividad positiva tenía un efecto negativo cuando la tarea se definía como seria y con refuerzos extrínsecos (Baas et al., 2008). Podemos hipotetizar para 
nuestro estudio que la inducción de alta afectividad positiva reforzará la creatividad, en particular si la inducción es de alta activación en comparación con un grupo en el que se induce una baja afectividad positiva, y si la tarea tiene una valencia positiva. Este efecto se dará además en los primeros 20 minutos de la inducción como sugiere el meta-análisis de Baas et al. 2008; véase Isen (2007) para ejemplos de efectos a los 45 minutos de afectividad inducida. La afectividad positiva debería inducir un rendimiento más creativo en tareas, así como ayudar a encontrar aspectos positivos o beneficios personales e interpersonales después de hechos de cambio vital. Un estudio longitudinal encontró que los sujetos resilientes sentían más emociones positivas después de hechos traumáticos colectivos y mostraban mayor crecimiento postestrés (Fredrickson, 2009).

\section{Afectividad positiva inducida, metas y ajuste}

Existe evidencia que hechos de cambio positivos extremos - como el enamoramiento intenso - se asocian tanto o más fuertemente que los hechos negativos al crecimiento después de la situación de cambio (Bilbao et al., 2013). En este estudio queremos contrastar que una alta afectividad positiva inducida se asociara a una evaluación más positiva de estos cambios. La reevaluación positiva es un antecedente del crecimiento post-estrés y se puede concebir a éste como una forma de creatividad - se encuentra lo positivo de algo negativo, se re-encuadra lo negativo como positivo o se cambia a una nueva perspectiva-. Como hemos mencionado anteriormente, las emociones positivas ayudan a darle significado a hechos estresantes y a reconstruir fines y propósitos en la vida (Fredrickson, 2009) y esto es de especial interés para nuestro estudio. Por ejemplo, podemos pensar que reforzar la afectividad positiva aumentará la percepción de ajuste y crecimiento después de un episodio emocional intenso -no solo negativo sino que también positivo - en este caso una experiencia de enamoramiento intenso sobre el que se pide que se evalúen los beneficios o el crecimiento personal e interpersonal asociado a él.

\section{Hipótesis}

En base a lo antes reseñado, nos hemos planteado como primera hipótesis que la alta afectividad positiva inducida conducirá a una mayor creatividad. Ésta se manifestará en la elaboración de poemas evaluados como más originales por jueces expertos e independientes, así como en narraciones de una experiencia emocional intensa más original y creativa. También inducirá una mayor percepción de cambios positivos después del episodio emocional intenso y las personas participantes en la muestra evaluaran que han alcanzado metas de ajuste más elevadas después de este suceso biográfico. Efectos similares pero menos intensos se esperan sobre rendimiento en creatividad verbal y gráfica, dado el tiempo de duración de la inducción. Finalmente, como segunda hipótesis, pensamos que será el grado de activación de emo- ciones positivas el proceso mediador o explicativo de la influencia de la manipulación en la creatividad.

\section{Método}

\section{Participantes}

Los participantes de este estudio fueron $N=57$ estudiantes de Psicología de la UPV/EHU, quienes respondieron una serie de escalas como parte de sus prácticas de un curso de grado. La muestra quedó constituida por 44 (78\% mujeres) y $12(22 \%)$ hombres, con un rango de edad entre 19 y 63 años $(M=24.57$ años, $D T=7.3)$.

\section{Procedimiento e Instrumentos}

Los participantes realizaron la tarea durante la práctica de un curso de grado en la Universidad del País Vasco. Completaron un cuadernillo de tareas en una sola sesión de una duración aproximada de $1 \mathrm{~h}$ y media. Indicaron edad, sexo y si además de estudiar, trabajaban.

La práctica del curso de Psicología incluía escalas con las cuales se buscaba medir las emociones sentidas al elaborar tres poemas, al narrar un episodio de enamoramiento intenso y al evaluar el ajuste y el crecimiento después del mismo. También se indagó sobre creatividad narrativa o verbal y gráfica mediante el PIC-A. Describimos a continuación qué tareas e instrumentos contestaron las personas que formaron parte de la muestra.

Haikus (Ivcevic, Brackett y Mayer, 2007; Lowenstein, 2010; Rodríguez, 1999). Se preguntó a las personas participantes si conocían este tipo de poemas y qué sabían sobre ellos -cabe aclarar que nadie de la muestra había escuchado hablar sobre los mismos- Durante 5-7 minutos se dio información gráfico-visual-auditiva alusiva sobre este tipo de poemas que finalizó con un video sobre la autenticidad creativa. A continuación se les dio una hoja de tarea con instrucciones para la realización de tres haikus, con ejemplos y criterios a cumplir para su realización (Ivcevic et al., 2007). Los sujetos fueron asignados al azar a la condición de alta afectividad positiva $(n=28)$ o de baja afectividad positiva $(n=$ 29). Un sujeto tenía datos incompletos, por lo que los análisis se hicieron sobre dos grupos de $n=28$. El primer haiku era común y se daba como estímulo para las dos condiciones la misma palabra. En la condición de alta afectividad positiva los participantes escribían un haiku sobre primavera y otro sobre verano. En la condición de baja afectividad positiva escribían un haiku sobre otoño y otro sobre invierno (en este orden).

Esta tarea fue evaluada de forma independiente por dos jueces expertos en haiku -profesores universitarios que redactan y leen regularmente estos poemas $(N=56)$. Se les solicitó que leyesen todos los poemas y que posteriormente evaluaran la originalidad de los mismos en base a una escala tipo Likert donde $1=$ nada original y $10=$ muy original. También se solicitó a jueces no expertos -doctorandos de psico- 
logía- que evaluaran novedad/originalidad y autenticidad de los haikus en base a una escala Likert donde 1=nada y 5=mucho. Se utilizó el método Consensual Asssesment y el promedio de las dos puntuaciones como indicador. La correlación entre ambos jueces expertos fue satisfactoria, Spearman Brown $r=.56, p<.01$, al igual que la de los jueces no expertos $r=.60, p<.01$ para novedad y $r=.70, p<$ .01 para autenticidad.

Escala de emociones positivas y negativas de Fredrickson (Páez et al., 2011) Al finalizar la tarea de los haikus, los participantes debían responder sobre las emociones sentidas al redactar el tercer poema. La escala de Fredrickson mide diez emociones positivas y diez negativas. La fiabilidad de esta escala fue satisfactoria $(N=56$, alfa $=.94)$ para emociones positivas y (al$\mathrm{fa}=.80)$ para emociones negativas. Esta variable se utilizó tanto para chequear el impacto de la manipulación como para ver si los sujetos en la condición de alta afectividad positiva informaban de más emociones positivas y menos negativas que los de baja afectividad positiva durante la narración.

Narración (Ivcevic et al., 2007). Seguidamente, los participantes debían escribir durante 10 minutos sobre una relación amorosa importante o un enamoramiento intenso que hubieran vivido. En la hoja de instrucciones se solicitaba "Por favor describe o narra una narración amorosa importante o un enamoramiento intenso que hayas vivido y responde las siguientes preguntas: 1.- Qué ocurría en tu vida en ese momento; 2.- ¿Qué emociones experimentaste?; 3.- ¿Qué es lo que hacía que esta experiencia fuera única o diferente a otras? y 4.- ¿Qué aprendiste de esta experiencia, cómo te cambió?"

Estas narraciones fueron evaluadas de forma independientes por jueces - profesores universitarios expertos en su ámbito y específicamente en trabajo con jóvenes y adolescentes-. Se solicitó a los jueces que tras leer todas las narraciones, evaluaran en una escala tipo Likert donde $1=$ nada a $5=$ mucho los siguientes 4 criterios: 1.- Novedad: Tomando en cuenta como se narra en general esta experiencia, ¿Cuanto difiere o es distinto el tema de esta historia de las otras? (...) Evalúe la novedad o carácter diferente y original de la historia, no la calidad de lo contado. 2.- Eficacia: En el contexto descrito por la historia, ifue la emoción una experiencia constructiva? (...) Evalúe si la experiencia fue globalmente adaptativa o al contrario no ayudó a la persona a adaptarse exitosamente al medio y a sus necesidades (...) 3.- Autenticidad: ¿la experiencia le pareció auténtica, verdadera, genuina (aunque sea poco creíble o muy imaginativa)? (...) Puntúe alto si la narración muestra autenticidad. 4.- Evaluación global de la creatividad emocional: Para la evaluación global tome en cuenta los 3 criterios anteriores (...) pero también tome en cuenta la complejidad de la historia, así como si la narración integra temas opuestos o en conflicto (...) Se utilizó el promedio de sus dos puntuaciones como indicador.

Las correlaciones entre los dos jueces fueron positivas y satisfactorias, Spearman Brown $r=.78, p<.001$ para novedad; y en menor medida Spearman Brown $r=.39$, $\mathrm{p}<.05$ para eficacia; $r=.40, p<.05$ para autenticidad y $r=.45, \mathrm{p}<$
.01 para creatividad emocional total. También se les solicitó a los jueces que evaluaran correspondencia de la relación (SI/NO) Spearman Brown $r=.84$ y vigencia positi$\mathrm{va/negativa} \mathrm{de} \mathrm{la} \mathrm{misma:} \mathrm{la} \mathrm{relación} \mathrm{terminó} \mathrm{en} \mathrm{forma} \mathrm{positi-}$ va/negativa; la relación continúa vigente (al día de la narración) $r=$ 92. La fiabilidad unificada de novedad, eficacia, autenticidad, y creatividad emocional total de los dos jueces fue satisfactoria $(a l f a=.78)$. Al final de la sesión, los participantes informaban lo que habían sentido durante la narración, utilizando para ello la escala de emociones positivas y negativas de Fredrickson antes descrita. La fiabilidad también fue satisfactoria para emociones positivas durante las narraciones $(N=56$, alfa $=.93)$ y para emociones negativas (alfa $=.78)$.

Escala de percepción de cambio o crecimiento después del hecho extremo (Post-Traumatic Growth Inventory-PTGI (Tedeschi y Calhoun, 1996), versión corta (Páez et al., 2011). Tras responder a las emociones sentidas durante la narración, los participantes debían evaluar los cambios percibidos ante el suceso impactante narrado -como resultado de procesos emocionales y cognitivos surgidos a raíz del enfrentar este hecho- a través de una escala de 8 ítems tipo Likert (de rango 1=nada, a 7=mucho); ejemplos de ítems son: Un crecimiento personal. (...); Aprender sobre las capacidades, habilidades y resistencia personal. (...); Mejorar el conocimiento sobre sí mismo y los otros. (...); Apreciar lo que se tiene y aprender las prioridades importantes en la vida (...); Crecimiento y desarrollo espiritual (...); Valorar el apoyo que las personas ofrecen). La escala mostró una buena fiabilidad ( $N$ $=56$, alfa $=.84)$.

Evaluación de las metas de regulación al finalizar el episodio (Páez, Martínez, Sevillano, Mendiburo y Campos, 2012). Se midió a través de 7 ítems que preguntaban en qué medida se había logrado p.e., disminuir la intensidad con la que vivió esta experiencia emocional (...); disminuir el displacer, molestia o desagrado (...); entender...la situación o conflicto; controlar la experiencia emocional de la situación; resolver el problema asociado la situación (...); manejar las relaciones con otras personas, cambiarlas para mejor (...); mantener o mejorar su imagen personal ante otras personas (...). La escala de respuesta fue tipo Likert de 1=cambiar poco o nada a $10=$ cambiar mucho. La fiabilidad de esta escala fue satisfactoria alfa $=.84$.

PIC-A (Artola et al., 2012). Las siguientes tareas realizadas, consistieron en dos juegos de asociación narrativa o verbal y gráfica. La prueba de imaginación creativa para adultos, evalúa la creatividad a través del uso que la persona hace de su imaginación y establece sus índices en base a variables como fluidez, flexibilidad y originalidad narrativa y gráfica.

En este estudio los participantes evaluaron sus respuestas en base a las indicaciones del manual, categorizándolas como allí se indica. Además dos jueces evaluaron aplicando el manual, las respuestas a la tarea de asociación de forma independiente. La fiabilidad de las medidas de fluidez, flexibilidad y originalidad auto y hetero evaluadas (total nueve estimaciones) fue muy satisfactoria alfa $=.96$. 
Dos jueces expertos -profesores con formación universitaria en pintura y en artes plásticas- evaluaron de forma independiente la originalidad con una escala tipo Likert donde $1=$ nada original a $10=$ muy original el juego gráfico. Los jueces recibieron todos los dibujos y se les pidió que los "leyeran" en conjunto antes de evaluar su originalidad individual. Se evaluó con el método Consensual Asssesment y se utilizó el promedio de sus dos puntuaciones como indicador. La correlación entre ambos jueces fue satisfactoria, Spearman Brown $r=.84, p<.001$. Esta fue una medida dependiente de rendimiento en creatividad.

Análisis de datos Se realizaron comparaciones de medias mediante Anovas y t-tests, así como se uso la $r$ punto biserial como prueba de contraste y estimación del tamaño del efecto (condición estímulo positivo $=2$, negativo $=1$ ). Una regresión múltiple contrastó el rol mediacional de la afectividad en el impacto de la manipulación sobre la creatividad estableciéndose como nivel de significación estadística $p<.05$, utilizando el paquete estadístico SPSS 15.0 para Windows.
Para estimar la mediación mediante el test de Sobel se usó el Excel de Beckstead (2009).

\section{Resultados}

Las diferencias de medias observadas entre los que redactaron poemas asociados a alta afectividad positiva versus baja afectividad positiva confirma el impacto de la manipulación (Tabla 1). Análisis de t-test mostraron que los sujetos de la condición positiva en comparación con los de la condición negativa informaron de más interés $(M=2.17, D T(1.25)$ versus $M=1.54, D T(1.07))$, más cercanía/amor $(M=2.24, D T$ (1.06) versus $M=1.64, D T(1.02))$, mas diversión $(M=2.21$, $D T$ (1.11) versus $M=1.32$, DT (1.15)) y mayor orgullo $(M=$ 2.34, DT (1.14) versus $M=1.25, D T(1.04))$. Informaban de menos tristeza $(M=.11, D T(.41)$ versus $M=.79, D T(1.16))$ - todas las $t$ superiores a 2.05, todas con $p<.05$. El resto de las emociones no mostraban diferencias significativas.

Tabla 1. Diferencias de medias en balanza de afectos y originalidad último haiku por afectividad inducida $(N=56)$.

\begin{tabular}{|c|c|c|c|c|c|}
\hline & \multicolumn{2}{|c|}{ Condición de baja afectividad positiva } & \multicolumn{2}{|c|}{ Condición de alta afectividad positiva } & \multirow[t]{2}{*}{$F$} \\
\hline & $M$ & DT & $M$ & DT & \\
\hline Balanza afectos Haiku & 1.18 & $(1.08)$ & 1.78 & $(1.10)$ & $4.19 *$ \\
\hline Jueces haikus & 5.43 & $(1.66)$ & 6.52 & $(1.75)$ & $6.05^{*}$ \\
\hline
\end{tabular}

$* p<.05 * * p<.01 * * * p<.001$

Nota: Jueces expertos independientes Haikus, rango 1-10

Además de inducir más emociones positivas y en menor medida menos emociones negativas, el haiku final en alta afectividad positiva fue evaluado como más original que el haiku final en baja afectividad positiva por los jueces expertos, confirmando que la alta afectividad positiva induce más rendimiento creativo (ver Tabla 1). El tamaño del efecto para balanza de afectos fue de $r(56)=.27$ y para originalidad $r$ $=.32$, ambos $p<.03$. Sin embargo, la manipulación experimental no tuvo ningún efecto en la evaluación de novedad y autenticidad de los haikus evaluada por los dos jueces no expertos. La mayor sensibilidad de las evaluaciones de los jueces expertos sugiere la superior validez de éstas.

\section{Alta/Baja afectividad positiva inducida y su impacto en las emociones, ajuste percibido y crecimiento personal}

La Tabla 2 muestra las diferencias entre los grupos respecto al impacto en las emociones, el crecimiento y ajuste percibido al finalizar el episodio según la condición, así como su impacto en los indicadores de rendimiento creativo.

Tabla 2. Diferencias de medias en balanza de afectos o emociones sentidas durante la narración, estimación del ajuste y del crecimiento post-estrés, originalidad narración, verbal y gráfica.

\begin{tabular}{|c|c|c|c|c|c|}
\hline & \multicolumn{2}{|c|}{ Condición baja afectividad positiva } & \multicolumn{2}{|c|}{ Condición alta afectividad positiva } & \multirow[t]{2}{*}{$F$} \\
\hline & $M$ & DT & $M$ & $D T$ & \\
\hline Balanza de afecto narración & 1.10 & $(1.48)$ & 1.85 & $(1.41)$ & $3.77 *$ \\
\hline Crecimiento (PTGI) & 5.03 & $(1.29)$ & 5.57 & $(0.94)$ & $3.20+$ \\
\hline Ajuste (MARS) & 5.52 & $(1.89)$ & 6.47 & $(1.74)$ & $3.79 *$ \\
\hline Jueces CE de la narración & 2.77 & $(0.33)$ & 3.11 & $(0.51)$ & $8.46^{* *}$ \\
\hline Jueces novedad de la narración & 2.29 & $(0.54)$ & 2.89 & $(0.71)$ & $12.47 * * *$ \\
\hline Creatividad verbal (PIC-A) & 99.5 & $(50.0)$ & 97.7 & $(51.6)$ & .017 \\
\hline Creatividad gráfica (PIC-A) & 4.29 & $(1.06)$ & 4.44 & $(1.46)$ & .018 \\
\hline Jueces originalidad gráfica & 11.3 & $(2.09)$ & 12.0 & $(2.04)$ & 1.55 \\
\hline
\end{tabular}

\section{$+p<.10 * p<.05 * * p<.01 * * * p<.001$}

Nota: Crecimiento rango 1-7; Ajuste rango 1-10; Jueces narración, rango 1-5; creatividad verbal, las puntuaciones son la suma de fluidez rango 0-38, flexibilidad 0-36 y originalidad 0-114; creatividad gráfica, rango 0-94 según manual. Jueces originalidad gráfica, rango 2-20.

La condición de alta afectividad positiva indujo una mejor balanza de afectos o a informar de más emociones posi- tivas que negativas al narrar el episodio de enamoramiento. Además, los jueces evaluaron como más creativas y novedo- 
sas las narraciones hechas en esta condición, $r=.37, p<.01$ y $r=.43, p<.01$. La balanza de afectos al escribir haikus se asoció a la balanza de afectos al narrar la experiencia $r=.34$, $p<.01$ aunque no al ajuste ni al crecimiento.

Los resultados con respecto al cambio personal muestran que las personas que habían contestado en la condición de alta afectividad positiva informaron haber experimentado mayor crecimiento personal $r(55)=.24, p<.05$ y mayor ajuste después del episodio $r=.26, p<.03$. En particular en la condición de alta afectividad positiva, en comparación con la de baja, se informó de mayores niveles de mejora del conocimiento sobre sí mismo y los otros y de desarrollo espiritual, y en grado moderado un mayor cambio en la apreciación de la vida, y cambios en las relaciones con otros. Con respecto a las metas de ajuste, en la condición de alta afectividad positiva se informó de mayor placer, mayor control y disminución de la intensidad que en la condición de baja afectividad positiva. Hay que señalar que las puntuaciones de crecimiento y ajuste eran altas, mostrando que las personas evaluaban positivamente la experiencia narrada. La media de crecimiento de la condición de baja afectividad positiva fue de 5 en una escala de 1 a 7 , frente a 5.6 en la condición de alta afectividad, y el del ajuste de 5.5 en la condición de baja frente a 6.5 en la de alta afectividad positiva en una escala de 1 a 10. Finalmente la manipulación no tuvo efectos sobre la creatividad verbal y grafica en ninguna de sus evaluaciones.

\section{Análisis mediacional}

La balanza de afectos al narrar se asoció al ajuste $r=.28$ y al crecimiento $r=.34$, ambas $p<.01$. Ajuste y crecimiento se asocian $r=.25, p<.01$ por lo que dada esta correlación se creó una sola variable dependiente unificada. Para contrastar la mediación de la balanza de afectos entre la manipulación y el ajuste y crecimiento, se calcularon regresiones entre la VD unificada sobre la manipulación, incluyendo en la última regresión la balanza de emociones vinculadas a las narraciones como mediadora (ver Figura 1).

Primero, confirmando que la manipulación influía la VD, la regresión de la puntuación total del crecimiento y ajuste en la manipulación -variable predictora o VI- fue significativa, $F(1,52)=5.29, p<.03$, Beta estandarizado $c=.30, t=2.30$ $p<.03$ explicando el $9 \%$ de la varianza.

Segundo, como ya hemos mencionado, la manipulación se asociaba a la variable mediadora: la asociación entre manipulación y balanza de afectos era positiva y significativa, $F(1,52)=3.8, p<.05$, Beta estandarizado $a=.26 . t=1.95 p<$ .05 explicando el $7 \%$ de la varianza.

Tercero, confirmando que la variable mediadora influía la VD, la regresión de la balanza de afectos durante la narración -como VI o predictora- sobre la puntuación total del crecimiento y el ajuste fue significativa, $F(1,52)=8.06, p<$ .01 , Beta estandarizado .36, $t=2.84, p<.007$, explicando el $13 \%$ de la varianza.

La regresión múltiple de la puntuación total del crecimiento y el ajuste como VD unificada en la manipulación variable predictora o VI- y en la balanza de afectos - variable mediadora- fue significativa, $F(2,51)=5.83, p<.006$, siendo la $R$ múltiple de .43 , explicando el $19 \%$ de la varianza.

\section{MANIPULACIÓN \\ $2=$ Primavera $/$ Verano \\ $1=$ Otoño/Invierno}

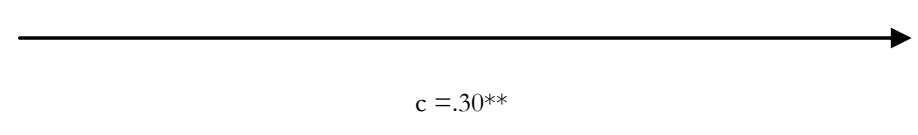

$\mathrm{c}=.30 * *$

\section{Ajuste (MARS) \\ Crecimiento} (PTGI)

BALANZA DE AFECTOS

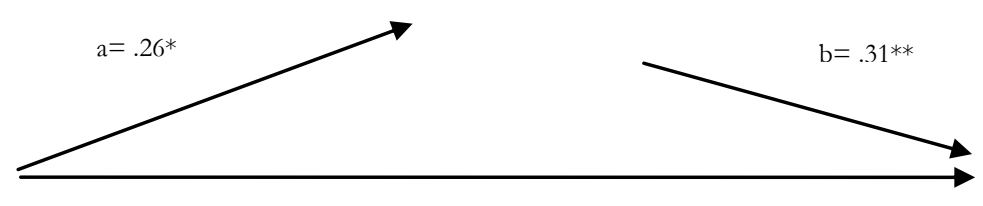

Ajuste (MARS)

MANIPULACIÓN

2=Primavera/Verano $1=$ Otoño $/$ Invierno

$$
c^{\prime}=23 \text { n.s. }
$$

Crecimiento

(PTGI)

Figura 1. Relaciones entre la manipulación, el crecimiento y ajuste, y la balanza de afectos. Análisis mediacional. Nota: a, b, c y c' betas estandarizados de la regresión; $*^{*} p<.001 ; * p<.05$.

La beta estandarizada de la manipulación baja de $\mathrm{c}=.30$ a $\mathrm{c}^{\prime}=.23$ y deja de ser significativa mientras que el beta de la balanza de afecto baja de $.36 \mathrm{a} b=.31$ y sigue siendo significativa. Esto indica una mediación sustancial y el test de Sobel fue de $Z=1.65, p<.05$ unilateral, confirmando que las emociones median entre la manipulación y el crecimiento y ajuste. El efecto indirecto fue de 0.40 y el $22 \%$ del efecto de la manipulación en el crecimiento y ajuste se deben a la mediación de la afectividad.

Análisis complementarios utilizando el procedimiento de Preacher y Hayes -que remuestrea aleatoriamente incrementando el $N$ y permite establecer intervalos de confianza para efectos directos e indirectos-, no entregaron información adicional. 


\section{Discusión}

Nuestro estudio examinó si los participantes en los que se indujo alta afectividad positiva presentaban mayor creatividad que aquellos en los que se indujo baja afectividad positiva. También se examinó si era el grado de activación de dichas emociones el proceso mediador o explicativo de la citada manipulación.

\section{Afectividad positiva inducida y creatividad percibida}

La inducción de alta afectividad positiva se mostró exitosa, generando más emociones positivas y menos negativas aunque con menor intensidad- en comparación con la condición de baja afectividad positiva, evaluadas mediante la escala de emociones positivas y negativas de Fredrickson.

La hipótesis se confirmó globalmente, ya que los sujetos en la condición de alta afectividad positiva informaron de más emociones positivas y menos negativas durante la elaboración de la narración. A su vez se confirmó que la alta afectividad positiva inducida condujo a una mayor creatividad. Específicamente se encontró en la condición de inducción de afectividad positiva, una evaluación de mayor originalidad del último poema evaluado por jueces expertos, una evaluación de mayor creatividad y novedad de las narraciones de la experiencia, una percepción de mayor crecimiento después de una experiencia amorosa intensa y mayor percepción que esta finalizó con un mejor ajuste o funcionalidad. Sin embargo, la hipótesis de la influencia de la afectividad positiva en la creatividad no se confirmó en los indicadores de creatividad verbal y gráfica de las tareas del PIC-A. Es decir, se obtuvo confirmación parcial de la hipótesis.

Globalmente los resultados confirman que las emociones y la afectividad positiva amplían los recursos cognitivos y ayudan a ser más creativos. Ahora bien, dado que se trata de auto-informes se podría concluir que la afectividad positiva aumenta las ilusiones y sesgos cognitivos positivos. En contra de esta idea, debemos destacar que aún aquellas personas en las que se indujo baja afectividad positiva muestran respuestas de carácter positivo, ya que informan de crecimiento medio-alto y de ajuste medio. Es decir, no perciben bajo crecimiento y ajuste. Una interpretación que nos parece más adecuada es que la inducción de una menor afectividad positiva y una ligera afectividad negativa provoca una visión más realista, pero no negativa, de las experiencias emocionales pasadas. Recordemos además que la redacción de poemas bajo el estímulo de baja afectividad positiva indujo una menor balanza de afectos pero no un predominio de emociones negativas. Los análisis específicos mostraron que se había inducido sentir más intensamente interés, diversión, amor/cercanía y orgullo en la condición de alta afectividad positiva y solo más tristeza en la condición de baja afectividad positiva siendo el nivel de tristeza bajo.

\section{Afectividad positiva inducida y rendimiento creativo}

Los efectos de la manipulación se han reflejado no solo en auto-informes de emoción, crecimiento y ajuste, sino que también en el rendimiento creativo del poema y de la narración. Respecto al rendimiento creativo podemos concluir que el efecto de la manipulación o influencia de la afectividad inducida se reflejo no solo en medidas de auto informe, sino también en evaluaciones independientes de productos creativos como la evaluación del poema y de la narración. Esto sugiere que los efectos de la afectividad positiva no solo inducen una percepción más positivista o una "visión más rosa", sino que también una mayor creatividad real.

Bass et al. (2008) han planteado que existe un efecto de congruencia entre el tipo de tarea y la influencia de la afectividad positiva. Nuestros resultados van en este sentido ya que la afectividad positiva refuerza la creatividad y reevaluación de un episodio de connotación mayoritariamente positivo. De hecho la mayoría de los episodios narrados $(82 \%)$ o seguían en la actualidad o habían terminado bien.

La manipulación experimental no tuvo ningún efecto en la evaluación de novedad y autenticidad de los haikus por jueces no expertos. Una explicación podría ser que el rango de respuestas dado para evaluar los haikus puede haber limitado la sensibilidad de la evaluación. Otra, que la evaluación de jueces no expertos tiene menor validez que la de jueces expertos, como ya mencionamos.

Isen (2007) plantea que las personas que han recibido afecto positivo inducido tienen un acceso más fácil al material positivo de la memoria y un mejor desempeño en las tareas importantes o interesantes, lo que se ha confirmado en nuestro estudio respecto a las dos primeras tareas. Sin embargo, la manipulación no tuvo efecto sobre la creatividad verbal y grafica evaluada por el PIC-A en ninguna de sus evaluaciones. Una posibilidad es que los indicadores de creatividad del PIC-A sean poco válidos - como critican algunos autores que descalifican las medidas de creatividad basadas en asociación de ideas (Romo, 2009). En contra de esto, encontramos toda la tradición de investigación con este instrumento (Artola et al., 2012). Otra posibilidad, que argumentaremos, es la limitación de efecto temporal de la inducción, que no estaría actuando en el momento de la respuesta al PIC-A. Siguiendo a Bass et al. (2008) planteamos al inicio de este trabajo que el efecto de la manipulación se daría en los primeros veinte minutos de inducción y esto se demuestra en nuestro estudio -que fue de 28 minutos- acercándonos más al planteamiento de Isen (2007).

\section{Afectividad inducida como mediador del efecto de la manipulación}

Los resultados confirman -como ya hemos argumentado-, que la manipulación tiene un efecto en el crecimiento y el ajuste, así como en la balanza de afectos. Además, esta balanza de afectos sentida al narrar se asoció al crecimiento después del cambio y a metas de ajuste. Las emociones sen- 
tidas al narrar, en particular las positivas, van ayudar a percibir mejor ajuste y más crecimiento personal, explicando alrededor del $9 \%$ de la varianza en las VD`s. Cuando la balanza de afectos se incluyó como predictora, eliminó la influencia significativa de la manipulación en el ajuste y crecimiento unificado. Podemos concluir que las emociones eliminan la influencia de la manipulación, mostrando una mediación total.

\section{Conclusiones}

Los hallazgos de este estudio confirman que la inducción de alta afectividad positiva conduce a una mayor creatividad. El afecto positivo impulsa al material positivo de la memoria, así las personas que se sienten felices tienen acceso a un amplio rango de pensamientos positivos y a una gran variedad de los mismos. Un modelo neurológico sobre la influencia de los afectos positivos en los procesos cognitivos sugiere que los resultados del afecto positivo son inducidos por el sistema de la dopamina en el cerebro (Isen, 2007). Es probable que la relación emoción-cognición sea el resultado del proceso evolutivo del cerebro consciente, y -quizá- motor de la misma. Algunos estudios sugieren que la emoción puede influir en la toma de conciencia de estímulos emocionales, cuando el nivel de atención es limitado. Los lóbulos frontales facilitan la visión de diferentes perspectivas y direcciones en la forma de pensamiento y de expresión, traduciéndose en fuente de creatividad (Salovey y Mayer, 1990).

Dado que el afecto positivo facilita procesos sociales y cognitivos, sería deseable que la inducción de afecto positivo se mantuviese y ejerciera su influencia sobre el pensamiento y el comportamiento, dado que el mismo se retroalimenta. De igual forma, se esperaría que las personas que se sienten felices retengan esos sentimientos o que lo experimenten más que otras personas (Isen, 2007). En este estudio, los participantes de la condición de alta afectividad positiva informaron de más emociones positivas durante la elaboración de la narración: de más interés, más cercanía/amor, más diversión y mayor orgullo; de una mayor percepción de cambios positivos después del episodio emocional intenso y evaluaron haber alcanzado metas de ajuste más elevadas que los sujetos en condición de baja afectividad positiva. Cuando las emociones sentidas se tomaban en cuenta, la influencia de la condición experimental de alta versus baja afectividad desaparecía, confirmando que la afectividad activada era el proceso explicativo de los efectos de la manipulación.

\section{Limitaciones e implicaciones en futuras investiga- ciones}

Como limitaciones al presente estudio podemos mencionar el no haber tenido una muestra de sujetos en condición neutra con la cual realizar una comparación, que potencialmente hubiese arrojado mayor luz en la interpretación de los resultados. Los argumentos a favor de incluir una condición neutra son, primero, porque era la comparación con la primera la que mostraba un efecto más fuerte (Bass et al., 2008). Segundo, porque los estudios ya desde los años 70 mostraban que el afecto positivo y moderado inducido servía como clave de recuperación para el material aprendido durante una sesión experimental, sin embargo eso no siempre es así en el caso de un bajo afecto positivo inducido, especialmente en la tristeza (Isen, 1999; Snyder y White, 1982). Es decir, la influencia cognitiva de la baja afectividad positiva fue menos consistente. Ahora bien, en contra de la importancia de una condición neutra, podemos mencionar, primero, que la comparación entre afectividad positiva y negativa encontró diferencias en dos dimensiones de la creatividad, y segundo, que los meta-análisis de regulación muestran que una condición neutra se transforma en positiva espontáneamente (Augustine y Hemenover, 2008). Es decir, consideramos que nuestro procedimiento probablemente tiene capacidad de detectar la influencia de la afectividad positiva inducida.

Un análisis post hoc de la potencia estadística (PE)- con el programa G*Power 3 de Faul, Erdfelder, Lang y Buchner, 2007-, mostró que ésta era de .50 para balanza de afectos y ajuste y de .43 para crecimiento, cuando se recomienda un poder estadístico de .80 . Con tamaños del efecto de .26 para balanza de afectos y ajuste y de .24 para crecimiento, con un alfa de .05 y un poder estadístico de .80 , se requieren 55 sujetos por condición en los dos primeros casos y 68 en el segundo para un poder estadístico de .80 . Es así que se pretende aumentar la $N$ para confirmar y/o mejorar la potencia y tener en cuenta los hallazgos registrados en el momento de hacer la réplica del mismo, planteando nuevas posibilidades, p.e., dividir el cuadernillo de tareas en dos días - a partir del PIC-A- con una inducción de afectividad positiva cada vez, teniendo en cuenta que según estudios las personas optimistas insisten más tiempo que las pesimistas en tareas que no tienen solución o con mayor dificultad y que los primeros dejan una tarea sin solución más pronto si hay tareas alternativas similares (Aspinwall y Richter, 1999)

Agradecimientos.-A todos/as los/as participantes y colaboradores por el compromiso y la dedicación tanto para las tareas como para con la evaluación. Este trabajo ha sido posible gracias a la Beca del Ministerio de Innovación y Ciencia (PSI2011-26315); beca del Gobierno Vasco al Grupo de Investigación (IT-666-13 y UFI 11/04) y la beca Predoctoral de la Universidad del País Vasco/Euskal Herriko Universitatea otorgada a la primera autora. 


\section{Referencias}

Augustine, A. A. \& Hemenover, S. H. (2008). On the relative effectiveness of affect regulation strategies: A meta-analysis. Cognition and Emotion, 23, 1181-1220. doi: 10.1080/02699930802396556

Artola, T., Barraca, J., Mosteiro, P., Ancillo, I., Poveda, B y Sánchez, N. (2012). PIC-A. Prueba de imaginación Creativa para adultos. Madrid, España: Tea.

Aspinwall, L. G. \& Richter, L. (1999). Optimism and self-mastery predict more rapid disengagement from unsolvable tasks in the presence of alternatives. Motivation and Emotion, 23, 221-245.

Baas, M., De Dreu, C. \& Nijstad, B. (2008). A Meta-Analysis of 25 Years of Mood-Creativity Research: Hedonic Tone, Activation, or Regulatory Focus? Psychological Bulletin, 134, 779-806.

Beckstead, J. (2009). Sobel test. Recuperado de personal.health.usf.edu/ibeckste/Sobel\%20Test.xls

Bilbao, Páez, da Costa y Martínez-Zelaya (2013). Cambio en creencias básicas y crecimiento post-estrés: un estudio transversal sobre el fuerte impacto de los hechos positivos sobre el bienestar. Terapia Psicológica, 31, 127-139.

Csikszentmihalyi, M. (2011). Creatividad. El fluir y la psicología del descubrimiento y la invención. Barcelona, España: Paidós.

Diener, E. (2009). The science of well-being. New York, NY: Springer.

Faul, F., Erdfelder, E., Lang, A.G. \& Buchner, A. (2007). G*Power 3: A flexible statistical power analysis program for the social, behavioral, and biomedical sciences. Behavior Research Methods, 39, 175-191. doi: 10.3758/BF03193146

Fernández-Berrocal, P. y Extremera, N. (2003). Facilitación y asimilación emocional de la inteligencia emocional en el contexto educativo: hallazgos científicos de sus efectos en el aula. Educación, 332, 97-116.

Fredrikson, B. (2009). Positivity. New York, NY: Crown Publishers.

Han, K. (2003). Domain-specificity of creativity in young children: How quantitative and qualitative data support it. Journal of Creative Behavior, 37, 117-142.

Hennessey, B. A. \& Amabile, T. M. (2010). Creativity. Annual Review of Psychology, 61, 569-598. doi: 10.1146/annurev.psych.093008.100416

Hammond, M. M., Neff, N. L., Farr, J.L., Schwall, A. R. \& Zhao, X. (2011). Predictors of Individual-Level Innovation at Work: A Meta-Analysis. Psychology of Aesthetics, Creativity, and the Arts Creativity, and the Arts, 5, 90105. doi: $10.1037 / \mathrm{a} 0018556$

Hunter, S. T., Bedell, K. E., \& Mumford, M. D. (2007). Climate for creativity: A quantitative review. Creativity Research Journal, 19, 69-90. doi: $10.1080 / 10400410709336883$

Isen, A. M. (2007). El afecto positivo como una fuente de potencial humano. En L.G. Aspinwall y U.M. Staudinger (Eds), Psicologia del potencial bumano (pp 247-268). Barcelona, España: Gedisa.

Isen, A. M. (1999). Positive affect and creativity. In S. W. Russ (Ed.), Affect, creative experience, and psychological adjustment (pp. 3-17). Philadelphia, PA: Brunel/Mazel.
Ivcevic, Z., Brackett, M. \& Mayer, J. (2007). Emotional Intelligence and Emotional Creativity. Journal of Personality, 75, 199-236. doi: 10.1111/j.1467-6494.2006.00437.x

Lowenstein, T. (2010). Haikus Clásicos. La mejor poesía japonesa. Barcelona, España: Blume.

Ma, H. H. (2009). The Effect Size of Variables Associated With Creativity: A meta-analysis. Creativity Research Journal, 21, 30-42.

Mirowsky, J. \& Ross, C.E. (2007). Creative work and health. Journal of Healt and Social Behavior, 48, 385-403. doi: 10.1177/002214650704800404

Oliveira, E., Almeida, L., Ferrándiz, C., Ferrando, M., Sainz, M. y Prieto, M. D. (2009). Test de pensamiento creativo de Torrance (TTCT): elementos para la validez de constructo en adolescentes portugueses. Psicother$m a, 21,562-567$.

Páez, D., Martínez, F., Sevillano, V., Mendiburo, A. y Campos, M. (2012). Medidas de estilos de regulación afectiva (MARS) ampliado en ira y tristeza. Psicotherma, 24, 249-254.

Páez, D., Vázquez, C., Bosco, S. Gasparre, A., Iraurgi, I. y Sezibera, V. (2011). Crecimiento post-estrés y post-traumático: posibles aspectos positivos y beneficiosos de la respuesta a los hechos traumáticos. En D. Páez, C. M. Beristain, J. L.González-Castro, N. Basabe y J. de Rivera (Eds.). Superando la violencia colectiva y construyendo cultura de paz. Madrid, España: Fundamentos.

Palfai, T. P. \& Salovey, P. (1993). The influence of depressed and elated mood on deductive and inductive reasoning. Imagination, Cognition and Personality, 13, 57-71. doi: 10.2190/FYYA-GCRU-J124-Q3B2

Rodríguez, F. (1999). El haiku japones. Historia y traducción. Madrid, España: Poesía Hiperión.

Romo, M. (2009). Psicología de la creatividad. Barcelona, España: Paidós.

Runco, M. A. (1999). Divergent thinking. In M.A. Runco y S.Pritzker (Eds.) Encyclopedia of Creativity. San Diego, SD: Academic Press

Salovey, P. \& Mayer, J. D. (1990). Emotional Intelligence. Imagination, Cognition and Personality, 9, 185-211. doi: 10.1017/CBO9780511806582.019

Scheier, M. F., Weintraub, J.K. \& Carver, C.S. (1986). Coping with stress: Divergent strategies of optimists and pessimist. Journal of Personality and Social Psychology, 51, 1257-1264. doi: 10.1037//0022-3514.51.6.1257

Snyder, M. \& White, E. (1982). Moods and memories: Elation, depression, and remembering the eents of one's life. Journal of Personality, 50, 149167.

Sternberg, R. J. \& Lubart, T. I. (1995). Defying the crowd: Cultivating creativity in a culture of conformity. New York, NY: Free Press

Tedeschi, R. G. \& Calhoun, L. G. (1996). The Posttraumatic Growth Inventory: measuring the positive legacy of trauma. J Trauma Stress, 9, 455-71. doi: $10.1007 / \mathrm{BF} 02103658$

(Artículo recibido: 14-05-2013; Revisado: 07-03-2014; Aceptado: 02-07-2014) 